\section{ABSTRACT}

Aim: The objectives are to compare responses of breast cancer $(\mathrm{BCa})$ treatment groups (chemotherapy, tamoxifen, and aromatase inhibitors (Als) to each other and a control regarding (a) subjective oral health, (b) oral health-related behaviors, (c) oral health-related concerns, and (d) communication with health care providers.

Methods: Survey data were collected from 140 postmenopausal BCa patients and 41 healthy postmenopausal control respondents.

Results: BCa patients reported on average more frequent mouth sores/ mucositis (5-point scale with $1=$ never: 1.63 vs. $1.14 ; p<.01)$, glossadynia $(1.60$ vs. $1.07 ; p<.01)$, xerostomia ( 2.48 vs. $1.40 ; p<.01)$, and dysgeusia ( 2.10 vs. $1.46 ; p<.01$ ) than the control respondents. Patients undergoing chemotherapy were more aware that cancer treatment can affect their oral health than patients on tamoxifen/Al $(93 \%$ vs. $55 \% / 56 \%$; $p<$ .001). BCa patients reported being more frequently informed by oncologists about oral health-related effects of cancer treatment than by dentists. Oncologists/nurses were more likely to communicate about oral health-related treatment effects with patients undergoing chemotherapy than patients on tamoxifen or Als. Few BCa patients perceived dentists as knowledgeable about cancer treatment-related oral concerns and trusted them less than oncologists. Conclusions: BCa treatments impact oral health. Low percentages of $\mathrm{BCa}$ patients had received specific information about impacts of $\mathrm{BCa}$ treatments on oral health from their dentists.

MAY WORDSF breast neoplasms oral health, post menopause, antiestrogen medication, chemotherapy

\title{
Oral health-related concerns, behavior, and communication with health care providers of patients with breast cancer: impact of different treatments
}

\author{
L. Susan Taichman, RDH, MS, MPH, PhD; ${ }^{*}$ Catherine H. Van Poznak, MD; ${ }^{2}$ \\ Marita R. Inglehart, Dr. phil. habil ${ }^{3}$
}

${ }^{1}$ Clinical Associate Professor, Department of Periodontics and Oral Medicine, School of Dentistry, University of Michigan, Ann Arbor, MI, USA; ${ }^{2}$ Associate Professor, Department of Internal Medicine, Hematology and Oncology Division, University of Michigan, Ann Arbor, MI, USA; ${ }^{3}$ Professor, Department of Periodontics and Oral Medicine, School of Dentistry \& Department of Psychology, College of Literature, Science \& Arts, University of Michigan, Ann Arbor, MI, USA.

*Corresponding author e-mail: hipolite@umich.edu

Spec Care Dentist 38(1): 36-45, 2018

\section{Introduction}

In 2014, the American Cancer Society predicted that nearly 270,000 women in the United States would develop breast cancer (BCa) in 2016 ${ }^{1}$; in addition to these new cases, the percentage of BCa survivors has increased over the years ${ }^{1}$ due to improved treatment approaches, making it crucial for dental care providers to be knowledgeable concerning the oral health-related consequences of different $\mathrm{BC}$ a treatments.

Depending on the cancer stage and the histologic and molecular profile of the tumor, ${ }^{2,3}$ surgical treatment can be combined with systemic adjuvant therapy to reduce the risk of developing distant metastases. ${ }^{4,5}$ Systemic therapies include chemotherapy and antiestrogen therapy. ${ }^{6}$ These therapies are considered either before or after surgery based on the patients' needs. Given that nearly $75 \%$ of BCa express estrogen and/or progesterone receptors ${ }^{7,8}$ the majority of $\mathrm{BCa}$ patients receive either tamoxifen or aromatase inhibitors (AIs) (e.g., anastrozole, exemestane, or letrozole ${ }^{9}$ ) on a long term basis (5-10 years) to decrease the risk of BCa recurrence. ${ }^{10}$

Oral health-related consequences of chemotherapy have been well documented. ${ }^{11-13}$ During conventional adjuvant chemotherapy treatments, $\mathrm{BCa}$ patients reported mucositis, taste disturbances, and increased xerostomia. ${ }^{14}$ Oral mucositis was present in approximately $40 \%$ of these patients, with nearly half requiring medical intervention, including modification of the cytotoxic cancer therapy. ${ }^{15}$ While extensive research concerning the oral complications of chemotherapy was conducted and even led to the development of guidelines for the management of oral mucositis, ${ }^{16}$ very few studies so far explored oral health complications of $\mathrm{BCa}$ treatments beyond the chemotherapy phase of cancer treatment.

Recent research showed that AIswhich severely diminish estrogen levels-affect BCa patients' periodontal health and reduce salivary flow, and thus impact their oral health-related quality of 
life. ${ }^{17-19}$ Hyposalivation can increase gingival inflammation and dental caries, and may also be responsible for an increased prevalence of oral dysesthesia and alterations in taste sensation. ${ }^{20,21}$ Many women with non-metastatic $\mathrm{BCa}$ on AIs receive bisphosphonates or other antiresorptives such as zolendric acid, ibandronate, or more recent, denosumab to prevent cancer treatment-induced bone loss. ${ }^{3}$ Osteonecrosis of the jaw is a rare but important adverse event associated most often with intravenous bisphosphonates or prolonged administration of bone inhibitors for the treatment of advanced cancer. $^{22,23}$

In addition, women using antiestrogen therapies may experience greater levels of depression, musculoskeletal symptoms, and fatigue, any of which may impact their ability to maintain optimal oral hygiene. ${ }^{24}$ However, relatively little is known about the oral healthrelated effects of $\mathrm{BCa}$ patients on tamoxifen. ${ }^{25}$ A comparison of the oral health-related concerns of patients treated with conventional chemotherapy, tamoxifen, or AIs would therefore clearly increase oncologists', oncology nurses,' and dental professionals' understanding of their role in the prevention, recognition, and management of oral complications of these therapies for $\mathrm{BCa}$ patients.

Research shows that so far oral problems were often underdiagnosed by physicians and not addressed by nurses, ${ }^{26}$ and that the implementation of an organized plan for oral care was relatively often overlooked by the oncology team. ${ }^{27,28}$ Likewise, a cross-sectional survey of dental hygienists in the state of Michigan showed that the majority of dental hygienists were not aware of oral health-related information about dental care for BCa survivors. ${ }^{25}$ For example, while $60 \%-80 \%$ of dental hygienists knew that mucosal changes, xerostomia, and increased risk for gingival tissue change were common oral complications of chemotherapy, only 10\%-12\% were aware that oral changes might occur when BCa survivors were treated with tamoxifen or AIs. ${ }^{25}$ Gaining a better understanding about which information
BCa patients treated with chemotherapy, tamoxifen, or AIs actually received or wanted to obtain from their oncology team or dental care provider could be crucial to advocate for better oral healthrelated support services.

Objective 1 of this study is to compare responses related to (a) subjective oral health, (b) oral health-related behaviors (i.e., tooth brushing, flossing, dental visits, and dental products used), and (c) oral health-related symptoms of $\mathrm{BCa}$ patients undergoing conventional chemotherapy, tamoxifen, or AIs treatment with the responses of postmenopausal control respondents. Objective 2 focuses on oral concern-related responses of the three groups of BCa patients after they had received a $\mathrm{BCa}$ diagnosis, and specifically on the responses of $\mathrm{BCa}$ patients on AIs concerning their oral health-related experiences in each stage of their previous and current cancer therapy. Objective 3 is to investigate how the three groups of BCa patients differed in their responses concerning their oral health-related communication with different healthcare providers.

\section{Methods}

\section{Study design and procedures}

This was a cross-sectional survey of women diagnosed with early stage $\mathrm{BCa}$ who received care at the University of Michigan Comprehensive Cancer Center (UMCCC) between June 2014 and June 2015. This study was reviewed and determined to be exempt by the Institutional Review Board at the University of Michigan prior to surveying participants (\#HUM00089395).

\section{Patient selection criteria}

An a priori power analysis with the program package G*Power 3.1.2 (http:// www.psycho.uni-duesseldorf.de/ abteilungen/aap/gpower3) was conducted to determine the sample size needed to have the power to test hypotheses concerning differences in the average responses of the three groups of $\mathrm{BCa}$ patients treated with conventional chemotherapy, tamoxifen, or AIs (e.g., anastrozole, exemestane, or letrozole) and the postmenopausal women in a control group. Assuming that we use a univariate analysis of variance for the comparison of the means of these four groups of respondents, that alpha $=.05$, the power $=.80$, and a large effect size $=$ 0.40 , a minimum of 19 subjects in each of the four groups would be needed. Data were collected from more than 19 respondents in each of the four groups.

$\mathrm{BCa}$ patients who were scheduled to receive treatment at the University of Michigan Breast Care Center, Ann Arbor, Michigan, were approached for inclusion in this study. A BCa patient was defined as a patient with a histologically confirmed diagnosis of non-metastatic cancer who was undergoing an active phase of adjuvant $\mathrm{BCa}$ treatment: conventional chemotherapy, or being treated with tamoxifen or AI therapy for at least 3 months. The recruitment of BCa patients occurred during their regularly scheduled medical oncology clinic appointments. Trained research assistants approached potential study subjects in the waiting area of the oncology clinics and informed them about the study.

Control group participants were postmenopausal women without a cancer diagnoses who were recruited from the University of Michigan Clinical Study website and at the University of Michigan-School of Dentistry. Patients in both groups were excluded if they had less than 20 natural teeth or if for any reason they were unable to understand the requirements of the study. Only postmenopausal BCa patients and controls were included for this analysis.

\section{Questionnaire}

The questionnaire was created by the authors from previously validated questionnaires ${ }^{19,27}$ and was pretested with a group of $20 \mathrm{BCa}$ volunteers from UMCCC. Based on the feedback from this pilot study, the survey was finalized and then administered.

The 54-item self-administered questionnaire assessed (1) respondents' socio-demographic characteristics and lifestyle factors (see Table 1); (2) 
Table 1. Background- and Hfestyle-related characteristics of the respondents in the four groups.

\begin{tabular}{|c|c|c|c|c|c|}
\hline Background characteristics & Chemotherapy $^{1} \mathrm{~N}=28$ & Tamoxifen $\mathrm{N}=52$ & Al $N=60$ & Control $N=41$ & All $\mathrm{BCa} N=140$ \\
\hline \multirow{2}{*}{ Age: Mean \pm SD Range } & $58.0 \pm 9.9$ & $56.3 \pm 8.3$ & $62.5 \pm 7.7$ & $66 \pm 9.4$ & $59.4 \pm 8.8$ \\
\hline & $32-78 b^{*}, c^{* * *}, g^{* * *}$ & $42-79 d^{* * *}, e^{* * *}$ & $45-78 f^{*}$ & $48-81 h^{* * *}$ & $32-79 i^{* * *}$ \\
\hline Married or living as married: Yes & $61 \% a^{* *}, g^{*}$ & $89 \% \mathrm{e}^{* * * *}$ & $77 \% f^{* * *}$ & $51 \% h^{* * *}$ & $78 \% i^{* * *}$ \\
\hline \multicolumn{6}{|l|}{ Ethnic/racial background: } \\
\hline - European American & $79 \%$ & $90 \%$ & $87 \%$ & $73 \%$ & $86 \%$ \\
\hline - African American & $18 \%$ & $4 \%$ & $7 \%$ & $20 \%$ & $8 \%$ \\
\hline - Asian American & $4 \%$ & $6 \%$ & $3 \%$ & $2 \%$ & $4 \%$ \\
\hline - Latina & $0 \%$ & $0 \%$ & $2 \%$ & $2 \%$ & $1 \%$ \\
\hline - Other & $0 \%$ & $0 \%$ & $2 \%$ & $3 \%$ & $1 \%$ \\
\hline \multicolumn{6}{|l|}{ Years of school: } \\
\hline$-<$ High school & $0 \%$ & $0 \%$ & $0 \%$ & $5 \%$ & $0 \%$ \\
\hline - High school & $17 \%$ & $10 \%$ & $8 \%$ & $24 \%$ & $10 \%$ \\
\hline - Some college & $13 \%$ & $25 \%$ & $12 \%$ & $34 \%$ & $18 \%$ \\
\hline - 2-yr college degree & $15 \%$ & $8 \%$ & $13 \%$ & $12 \%$ & $11 \%$ \\
\hline - 4-yr college degree & $28 \%$ & $19 \%$ & $20 \%$ & $10 \%$ & $23 \%$ \\
\hline - Graduate training & $10 \%$ & $39 \% \mathrm{e}^{*}$ & $47 \% f^{* * *}$ & $15 \% h^{* *}$ & $39 \% i^{* * *}$ \\
\hline \multicolumn{6}{|l|}{ Current employment status: } \\
\hline - Working full time & $43 \%$ & $33 \%$ & $38 \%$ & $10 \%$ & $31 \%$ \\
\hline - Working part time & $7 \%$ & $19 \%$ & $3 \%$ & $17 \%$ & $12 \%$ \\
\hline - Not employed & $18 \%$ & $23 \%$ & $27 \%$ & $24 \%$ & $24 \%$ \\
\hline - Retired & $25 \%$ & $23 \%$ & $30 \%$ & $34 \%$ & $28 \%$ \\
\hline - Other & $7 \% \mathrm{c}^{* * *}$ & $2 \% \mathrm{e}^{*}$ & $2 \% f * *$ & $15 \% \mathrm{~h}^{*}$ & $5 \% i^{* *}$ \\
\hline \multicolumn{6}{|l|}{ Yearly combined family income: } \\
\hline$-\$ 1-\$ 29,999$ & $11 \%$ & $11 \%$ & $2 \%$ & $15 \%$ & $7 \%$ \\
\hline$-\$ 30,000-49,999$ & $11 \%$ & $6 \%$ & $13 \%$ & $13 \%$ & $10 \%$ \\
\hline$-\$ 50,000-79,999$ & $26 \%$ & $26 \%$ & $32 \%$ & $25 \%$ & $29 \%$ \\
\hline - Over $\$ 80,000$ & $52 \% c^{* *}$ & $57 \% \mathrm{e}^{* *}$ & $54 \% f^{* * *}$ & $46 \% h^{* * *}$ & $55 \% i^{* * *}$ \\
\hline Dental insurance: Yes & $82 \% c^{*}$ & $75 \%$ & $80 \% f^{*}$ & $61 \%$ & $79 \% i^{*}$ \\
\hline \multicolumn{6}{|l|}{ Lifestyle-related behaviors } \\
\hline Smoking: Yes & $4 \% \mathrm{~g}^{*}$ & $2 \% e^{* * *}$ & $2 \% f^{* * *}$ & $12 \%$ & $2 \% i^{*}$ \\
\hline Alcohol: Yes & $67 \% \mathrm{~g}^{* * *}$ & $64 \% \mathrm{e}^{* *}$ & $78 \% f^{* *}$ & $32 \% h^{* * *}$ & $71 \% i^{* * *}$ \\
\hline \multicolumn{6}{|c|}{$\begin{array}{l}\text { Note: *p<.05; ** } p<.01 ;{ }^{* * *} p<.001 \text {. } \\
\text { "Significant differences between specific groups are indicated as follows: } \\
\text { a: Significant difference of the "chemotherapy group" (C) versus the "tamoxifen group" (T) responses. } \\
\text { b: Significant difference of the "chemotherapy group" versus the "Al group" (A) responses. } \\
\text { c: Significant difference of the "chemotherapy group" versus the "control group" (CG) responses. } \\
\text { d: Significant difference of the "tamoxifen group" versus the "Al group" responses. } \\
\text { e: Significant difference of the "tamoxifen group" versus the "control group" responses. } \\
\text { f: Significant difference of the "Al group" versus the "control group" responses. } \\
\text { g: Significant difference of the three treatment groups (C vs. T vs. A) responses. } \\
\text { h: Significant difference of the four groups (C vs. T vs. A vs. CG). } \\
\text { i: Significant difference of the three BCa groups COMBINED versus the "CG" responses. }\end{array}$} \\
\hline
\end{tabular}

subjective oral health indicators as well as oral health-related behaviors (i.e., frequency of brushing and flossing, dental visits, and dental products used) (see Table 2); and (3) oral-health symptoms including the presence or absence of oral problems such as xerostomia, dysgeusia, and the perception of oral health impacts (see Table 3 and Figure 1). In addition, BCa patients filled out a survey section on perceptions of the effects of cancer on oral health and their communication with their oncology and dental care providers about these issues (see Tables 4 and 5). 
Table 2. Percentages of subjective oral health and oral health-related behavior responses by group of respondents.

\begin{tabular}{|c|c|c|c|c|c|}
\hline Subjective oral health & Chemotherapy $^{1} \mathrm{~N}=28$ & Tamoxifen $\mathbf{N}=52$ & $\mathrm{Al} \mathrm{N}=60$ & Control $N=41$ & All $\mathrm{BCa} N=140$ \\
\hline $\begin{array}{l}\text { Health of teeth': } \\
\text { - Mean (SD) } \\
\text { - Range }\end{array}$ & $\begin{array}{c}3.21 \\
(.738) \\
2-4\end{array}$ & $\begin{array}{c}3.65 \\
(1.08) \\
1-5 d^{* * *}\end{array}$ & $\begin{array}{l}3.43 \\
(.890) \\
1-5 f^{*}\end{array}$ & $\begin{array}{c}2.73 \\
(.895) \\
1-5 h^{* * *}\end{array}$ & $\begin{array}{c}3.47 \\
(.917) \\
1-5 i^{* * *}\end{array}$ \\
\hline $\begin{array}{l}\text { Health of gums²: } \\
\text { - Mean (SD) } \\
\text { - Range }\end{array}$ & $\begin{array}{c}3.36 \\
(.911) \\
2-5\end{array}$ & $\begin{array}{c}3.48 \\
(.980) \\
1-5\end{array}$ & $\begin{array}{c}3.40 \\
(.785) \\
2-5\end{array}$ & $\begin{array}{c}2.93 \\
(.985) \\
1-5 h^{*}\end{array}$ & $\begin{array}{c}3.42 \\
(.882) \\
1-5 i^{* *}\end{array}$ \\
\hline \multicolumn{6}{|l|}{ Amount of saliva: } \\
\hline $\begin{array}{l}\text { - Too little } \\
\text { - Too much } \\
\text { - Perfect amount of saliva }\end{array}$ & $\begin{array}{c}21 \% \\
4 \% \\
75 \% a^{*}, b^{* *}, g^{*}\end{array}$ & $\begin{array}{c}27 \% \\
0 \% \\
73 \%\end{array}$ & $\begin{array}{c}38 \% \\
0 \% \\
62 \% \mathrm{~d}^{* * *}\end{array}$ & $\begin{array}{c}20 \% \\
7 \% \\
73 \% h^{* * *}\end{array}$ & $\begin{array}{c}31 \% \\
1 \% \\
68 \% i^{*}\end{array}$ \\
\hline \multicolumn{6}{|l|}{ Oral health-related behavior } \\
\hline \multicolumn{6}{|l|}{ Frequency of brushing: } \\
\hline $\begin{array}{l}\text { - Never/rarely } \\
\text { - Nearly every day } \\
\text { - Every day } \\
\text { - More than once a day }\end{array}$ & $\begin{array}{c}0 / 0 \% \\
0 \% \\
21 \% \\
79 \% \\
\end{array}$ & $\begin{array}{c}0 / 0 \% \\
4 \% \\
17 \% \\
79 \%\end{array}$ & $\begin{array}{c}0 / 0 \% \\
2 \% \\
26 \% \\
72 \% \\
\end{array}$ & $\begin{array}{c}2 / 2 \% \\
11 \% \\
22 \% \\
63 \% \\
\end{array}$ & $\begin{array}{c}0 / 0 \% \\
2 \% \\
22 \% \\
76 \% i^{* *}\end{array}$ \\
\hline \multicolumn{6}{|l|}{ Frequency of flossing: } \\
\hline $\begin{array}{l}\text { - Never } \\
\text { - Rarely } \\
\text { - Nearly every day } \\
\text { - Every day } \\
\text { - More than once a day }\end{array}$ & $\begin{array}{c}4 \% \\
18 \% \\
32 \% \\
36 \% \\
10 \% \\
\end{array}$ & $\begin{array}{c}2 \% \\
24 \% \\
31 \% \\
31 \% \\
12 \% \\
\end{array}$ & $\begin{array}{c}0 \% \\
22 \% \\
45 \% \\
30 \% \\
3 \% \\
\end{array}$ & $\begin{array}{c}7 \% \\
10 \% \\
29 \% \\
39 \% \\
15 \% \\
\end{array}$ & $\begin{array}{c}1 \% \\
22 \% \\
37 \% \\
32 \% \\
8 \% i^{*}\end{array}$ \\
\hline \multicolumn{6}{|l|}{ Last dental visit: } \\
\hline $\begin{array}{l}- \text { Never } \\
->13 \text { months ago } \\
-7-12 \text { months ago } \\
-4-6 \text { months ago } \\
-0-3 \text { months ago }\end{array}$ & $\begin{array}{l}0 \% \\
4 \% \\
14 \% \\
36 \% \\
46 \% \\
\end{array}$ & $\begin{array}{c}0 \% \\
8 \% \\
12 \% \\
24 \% \\
56 \% \\
\end{array}$ & $\begin{array}{c}0 \% \\
7 \% \\
8 \% \\
30 \% \\
55 \% \\
\end{array}$ & $\begin{array}{c}0 \% \\
20 \% \\
6 \% \\
32 \% \\
44 \%\end{array}$ & $\begin{array}{c}0 \% \\
6 \% \\
11 \% \\
30 \% \\
53 \% i^{*}\end{array}$ \\
\hline \multicolumn{6}{|l|}{ Oral health-related behavior } \\
\hline \multicolumn{6}{|c|}{ Products requested from dentist for: } \\
\hline $\begin{array}{l}\text { - Mucositis } \\
\text { - Halitosis } \\
\text { - Xerostomia } \\
\text { - Glossadynia }\end{array}$ & $\begin{array}{c}4 \% \\
0 \% \\
18 \% b^{*}, c^{*}, g^{* *} \\
0 \%\end{array}$ & $\begin{array}{c}0 \% \\
2 \% \\
20 \% \mathrm{e}^{*} \\
4 \%\end{array}$ & $\begin{array}{c}0 \% \\
3 \% \\
29 \% f^{*} \\
2 \%\end{array}$ & $\begin{array}{c}0 \% \\
2 \% \\
7 \% \mathrm{~h}^{*} \\
0 \%\end{array}$ & $\begin{array}{c}2 \% \\
3 \% \\
24 \% i^{*} \\
3 \%\end{array}$ \\
\hline \multicolumn{6}{|c|}{$\begin{array}{l}\text { Note: "p<.05; }{ }^{* *} p<.01 ;{ }^{* *} p<.001 . \\
\text { 'Significant differences between specific groups are indicated as follows: } \\
\text { a: Significant difference of the "chemotherapy group" (C) versus the "tamoxifen group" (T) r } \\
\text { b: Significant difference of the "chemotherapy group" versus the "Al group" (A) responses. } \\
\text { c: Significant differences of the "chemotherapy group" versus the "control group" (CG) resp } \\
\text { d: Significant difference of the "tamoxifen group" versus the "Al group" responses. } \\
\text { e: Significant difference of the "tamoxifen group" versus the "control group" responses. } \\
\text { f: Significant difference of the "Al group" versus the "control group" responses. } \\
\text { g: Significant difference of the three treatment groups (C vs. T vs. A) responses. } \\
\text { h: Significant difference of the four groups (C vs. T vs. A vs. CG). } \\
\text { i: Significant difference of the three BCa groups COMBINED versus the "CG" responses. } \\
\text { 2The answers range from } 1=\text { "poor" to } 5 \text { = "excellent." }\end{array}$} \\
\hline
\end{tabular}

\section{Statistical analysis}

The data were entered into an Excel spread sheet (Microsoft), and then examined for accuracy and completeness. They were then imported into SPSS (IBM SPSS Statistics for Windows, Version
22.0. Armonk, NY: IBM Corp; Released 2013). Descriptive statistics such as frequency distributions, percentages, means, and standard deviations were computed to provide an overview of the findings. Univariate analyses of variance were used to compare the mean answers of BCa survivors being treated with chemotherapy, tamoxifen or AIs, and the control subjects; independent sample $t$-tests were used to compare the mean answers of the combined group of $\mathrm{BCa}$ 
Table 3. Participant responses concerning the average frequency of oral symptoms reported within the last 3 months.

\begin{tabular}{|c|c|c|c|c|c|}
\hline In the last 3 months have you had': & Chemotherapy $^{1} \mathrm{~N}=28$ & Tamoxifen $\mathrm{N}=52$ & Al $N=60$ & Control N = 41 & All $\mathrm{BCa} N=140$ \\
\hline Mouth sores or mucositis & $2.14 \pm 1.09 \mathrm{~b}^{* * *}, \mathrm{c}^{* * *}, \mathrm{~g}^{* * *}$ & $1.65 \pm 1.18 \mathrm{e}^{* *}$ & $1.36 \pm 1.18$ & $1.14 \pm 0.42 h^{* * *}$ & $1.63 \pm 1.04 i^{* *}$ \\
\hline Teeth sensitive to hot, cold, or sweets & $1.55 \pm 1.01 a^{*}, c^{*}$ & $2.25 \pm 1.39$ & $1.93 \pm 1.20$ & $2.12 \pm 1.14$ & $1.98 \pm 1.26$ \\
\hline Glossadynia & $2.11 \pm 1.33 a^{* * *}, c^{* * *}, b^{* * *}, g^{* * *}$ & $1.40 \pm 0.86 \mathrm{e}^{* * *}$ & $1.43 \pm 0.96 f^{*}$ & $1.07 \pm 0.34 h^{* *}$ & $1.60 \pm 1.04 i^{* *}$ \\
\hline Mouth feels dry & $2.53 \pm 1.42 \mathrm{c}^{*}$ & $2.31 \pm 1.44$ & $2.60 \pm 1.48 f^{* *}$ & $1.70 \pm 1.16 h^{*}$ & $2.48 \pm 1.46 i^{* *}$ \\
\hline Change in taste & $3.57 \pm 1.67 a^{* * *}, c^{* * *}, b^{* * *}, g^{* * *}$ & $1.84 \pm 1.45$ & $1.62 \pm 1.20$ & $1.46 \pm 0.80 h^{* * *}$ & $2.10 \pm 1.57 i^{* *}$ \\
\hline Bleeding gums & $1.50 \pm 1.00$ & $1.78 \pm 0.99$ & $1.60 \pm 0.96$ & $1.84 \pm 0.94$ & $1.65 \pm 0.98$ \\
\hline Aching in mouth, teeth, or jaw & $1.50 \pm 0.96$ & $1.42 \pm 0.76 e^{*}$ & $1.57 \pm 1.05$ & $1.82 \pm 1.11$ & $1.50 \pm 0.94$ \\
\hline Bad breath & $1.67 \pm 1.09$ & $1.88 \pm 0.96$ & $1.95 \pm 1.11$ & $2.02 \pm 0.97$ & $1.87 \pm 1.05$ \\
\hline \multicolumn{6}{|c|}{$\begin{array}{l}\text { Note: "p<.05; ** } p<.01 ;{ }^{* * *} p<.001 \text {. } \\
\text { "Significant differences between specific groups are indicated as follows: } \\
\text { a: Significant difference of the "chemotherapy group" (C) versus the "tamoxifen group" (T) responses. } \\
\text { b: Significant difference of the "chemotherapy group" versus the "Al group" (A) responses. } \\
\text { c: Significant differences of the "chemotherapy group" versus the "control group" (CG) responses. } \\
\text { e: Significant difference of the "tamoxifen group" versus the "control group" responses. } \\
\text { f: Significant difference of the "Al group" versus the "control group" responses. } \\
\text { g: Significant difference of the three treatment groups (C vs. T vs. A) responses. } \\
\text { h: Significant difference of the four groups (C vs. T vs. A vs. CG). } \\
\text { i: Significant difference of the three BCa groups COMBINED versus the "CG" responses. } \\
\text { "Answers range from } 1=\text { "Never", } 2 \text { = "Hardly ever", } 3 \text { = "On occasion", } 4 \text { = "Fairly often" to } 5=\text { "Very }\end{array}$} \\
\hline
\end{tabular}

patients and of the control group respondents. In addition, post hoc comparisons were conducted to compare the responses of sub groups of respondents, namely "chemotherapy group" versus "tamoxifen group" responses, "chemotherapy group" versus "AI group" responses, "tamoxifen group" versus "AI

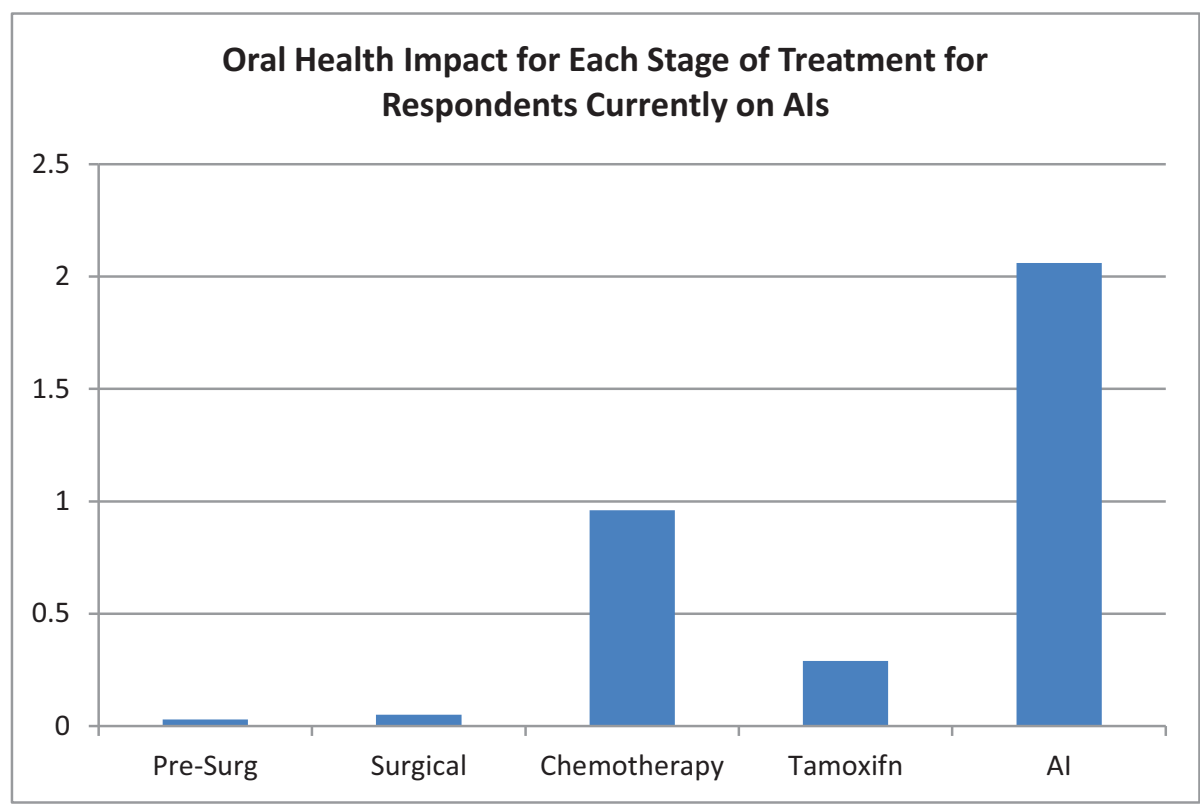

Figure 1. Respondents currently on Al: oral health impacts in each stage of treatment. Oral health impact was assessed by adding 1 point for reporting to have had mouth sores or mucositis; teeth sensitive to hot, cold, or sweets; glossadynia; dry mouth; change in taste; bleeding gums; aching in mouth, teeth, or jaw; and bad breath. The scores can range from 0 to 8 ( $8=$ highest impact).

group" responses, responses of the three treatment groups, and responses of each treatment group versus the control group. Chi-squared tests were used to test relations between categorical variables. The significance level was set at $p<.05$.

\section{Results}

A total of 300 patients with early stage $\mathrm{BCa}$ receiving either adjuvant chemotherapy or endocrine therapy (tamoxifen or AIs) for at least 3 months for $\mathrm{BCa}$ were approached for inclusion. Of these women, 263 agreed to participate (87\%). The current analyses were restricted to 181 women who were postmenopausal and had completed questionnaires (65\%). Data were collected from 28 BCa patients receiving adjuvant chemotherapy, 52 patients being treated with tamoxifen, 60 women being treated with AIs, and 41 women in a control group without any cancer diagnoses. Table 1 provides an overview of the background characteristics (a) for the four groups (3 cancer groups and the control group) as well as (b) for the combined group of 


\section{Table 4. Percentages of oral health concerns responses of BCa respondents.}

Oral concern-related responses: \% Yes

Problems with teeth or gums since diagnosis

Do you believe your oral health problem is related to your cancer treatment?

Are you aware that cancer treatment may affect oral health?

Who told you that cancer treatment may affect oral health?

- Primary care

- Nurse practitioner

- Oncologist

- Dental provider

- Other

- No one

Was there a time when you wanted to visit your dentist but could not because of your BCa treatment?

\begin{tabular}{|c|c|c|}
\hline Chemotherapy $^{1} \mathrm{~N}=\mathbf{2 8}$ & Tamoxifen $\mathrm{N}=52$ & Al $N=60$ \\
\hline $21 \%$ & $20 \%$ & $25 \%$ \\
\hline $30 \% a^{* * *}$ & $23 \%$ & $30 \%$ \\
\hline $93 \% a^{* *}, b^{* * *}$ & $55 \%$ & $56 \% \mathrm{~d}^{* * *}$ \\
\hline $\begin{array}{c}0 \% \\
7 \% \\
60 \% a^{* * * *}, b^{* * *} \\
25 \% \\
6 \% \\
2 \% a^{* * *}, b^{* * *}\end{array}$ & $\begin{array}{c}0 \% \\
5 \% \\
34 \% \\
24 \% \\
2 \% \\
35 \%\end{array}$ & $\begin{array}{c}0 \% \\
10 \% \\
32 \% \mathrm{~d}^{* * *} \\
25 \% \\
4 \% \\
44 \%\end{array}$ \\
\hline $29 \%$ & $22 \%$ & $24 \%$ \\
\hline
\end{tabular}

Point of treatment at which your dentist discussed impacts of cancer treatment on oral health:

\section{- Presurgical}

- Chemotherapy

- Radiation

- Antiestrogen

- Never discussed

- Have not visited dentist since diagnosis

Note: ${ }^{*} p<.05 ;{ }^{* *} p<.01 ;{ }^{* * *} p<.001$.

'Significant subgroup differences are indicated as follows:

a: Significant difference of the "chemotherapy group" versus the "tamoxifen group" responses.

b: Significant difference of the "chemotherapy group" versus the "Al group" responses.

c: Significant difference of the "tamoxifen group" versus the "Al group" responses.

$\mathrm{d}$ : Significant difference of the three treatment groups responses.

\section{Table 5. Responses related to oral health-related patient-provider interactions.}

\begin{tabular}{|c|c|c|c|}
\hline Providers' oral health-related behaviors & Chemotherapy $^{1} \mathrm{~N}=28$ & Tamoxifen $\mathrm{N}=52$ & Al N $=60$ \\
\hline Oncologist/nurse asked about your oral health? Yes & $83 \% a^{* *}, b^{* *}, d^{* * *}$ & $32 \%$ & $33 \%$ \\
\hline Oncologist/nurse asked about mouth sores or dry mouth? Yes & $92 \% a^{* *}, b^{* *}, d^{* * *}$ & $36 \% c^{*}$ & $44 \%$ \\
\hline \multicolumn{4}{|l|}{ Dental providers asked if oral health has changed during cancer treatment: } \\
\hline $\begin{array}{l}\text { - Yes } \\
\text { - No } \\
\text { - "I have not been to dentist since diagnosis" }\end{array}$ & $\begin{array}{c}23 \% \\
31 \% \\
46 \% a^{* *}, b^{* *}, d^{*}\end{array}$ & $\begin{array}{c}50 \% \\
40 \% \\
10 \% c^{* *}\end{array}$ & $\begin{array}{l}44 \% \\
48 \% \\
8 \%\end{array}$ \\
\hline Patient oral health-related responses to providers: \% Yes & Chemotherapy $\mathrm{N}=28$ & Tamoxifen $\mathrm{N}=52$ & Al $N=60$ \\
\hline Confident to ask medical provider about oral problems? & $94 \%$ & $96 \%$ & $98 \%$ \\
\hline Confident to ask nurse about oral problems? & $94 \% b^{*}$ & $88 \%$ & $98 \%$ \\
\hline Comfortable asking dental provider about oral problems? & $86 \% b^{*}, d^{*}$ & $82 \% c^{*}$ & $96 \%$ \\
\hline Patients' perception of provider's oral-health knowledge & Chemotherapy $\mathrm{N}=28$ & Tamoxifen $\mathrm{N}=52$ & Al N $=60$ \\
\hline Oncologist/nurse is knowledgeable about oral problems? Yes & $100 \% a^{*}, b^{*}$ & $89 \%$ & $95 \%$ \\
\hline $\begin{array}{l}\text { Dental provider is knowledgeable about oral health needs during my cancer treat- } \\
\text { ment? Yes }\end{array}$ & $70 \%$ & $65 \%$ & $53 \%$ \\
\hline $\begin{array}{l}\text { Patients' confidence that dental provider can answer oral health/cancer treatment- } \\
\text { related concerns: Yes of confident/very confident }\end{array}$ & $65 \% d^{*}$ & $56 \% c^{*}$ & $52 \%$ \\
\hline \multicolumn{4}{|c|}{$\begin{array}{l}\text { Note: }{ }^{*} p<.05 ;{ }^{* *} p<.01 ;{ }^{* * *} p<.001 . \\
\text { 'Significant subgroup differences are indicated as follows: } \\
\text { a: Significant difference of the "chemotherapy group" versus the "tamoxifen group" responses. } \\
\text { b: Significant difference of the "chemotherapy group" versus the "Al group" responses. } \\
\text { c: Significant difference of the "tamoxifen group" versus the "Al group" responses. } \\
\text { d: Significant difference of the three treatment groups responses. }\end{array}$} \\
\hline
\end{tabular}


BCa patients versus the control respondents. BCa patients were on average about 7 years younger than the control subjects (59.4 years vs. 66 years; $p<.001$ ) and were more likely to be married or living as married $(p<.001)$ than the control respondents; they were significantly better educated $(p<.05)$, were more likely to work full time $(p<.01)$, and had a higher yearly combined family income $(p<.001)$. BCa patients were also less likely to smoke $(p<.05)$, but more likely to consume alcohol $(p<.001)$ compared to the control subjects. When comparing the three BCa groups, women undergoing chemotherapy were younger, smokers, and less likely to be married than either Tamoxifen or AI participants. Also women on AI treatment were significantly more likely to consume alcohol as compared to women undergoing chemotherapy or using tamoxifen (78\% vs. $67 \%$ vs. $64 \% ; p<.001$ ).

Table 2 provides an overview of the responses related to respondents' subjective oral health and oral healthrelated behavior. On average, the control group reported poorer mean health of teeth (scale from $1=$ poor to $5=$ excellent: 2.73 vs. $3.47 ; p<.001$ ) and poorer mean health of their gums (2.93 vs. 3.42; $p<.05)$ than the three groups of $\mathrm{BCa}$ patients combined. BCa patients were more likely to report having too little saliva than the control group (31\% vs. $20 \% ; p<.05)$. However, when the three BCa patient groups were compared in regard to their amount of saliva, the AI group was significantly more affected with xerostomia than the tamoxifen and the chemotherapy groups.

Compared to the control group subjects, BCa patients were more likely to brush more than once a day $(p<.01)$, but less likely to floss frequently $(p<.05)$. BCa patients were more likely to have had a dental visit in the recent past $(53 \%$ vs. $44 \%, p<.05)$ than the control respondents. AI users were more likely to have asked their dental provider for products related to xerostomia( $29 \%$ vs. $18 \%$ vs. $20 \%$; $p<.01)$ than chemotherapy or tamoxifen respondents.
Table 3 provides an overview of the average frequency of oral symptoms within the last 3 months by type of cancer treatment versus being a control group respondent. These data show that $\mathrm{BCa}$ patients reported more frequent problems with mouth sores/mucositis (scale from 1 = "never" to 5 = "very often": 1.63 vs. $1.14 ; p<.01$ ), oral dysesthesia ( 1.60 vs. $1.07 ; p<.01$ ), with xerostomia ( 2.48 vs. $1.70 ; p<.01$ ), and having experienced dysgeusia (2.10 vs. $1.46 ; p<.01)$ than the control group respondents. In addition, this table also shows that respondents in the three $\mathrm{BCa}$ patient sub groups differed in the average frequencies with which they reported some of these symptoms. For example, while chemotherapy group members did not differ significantly from tamoxifen group members in the average frequency of mouth sores/mucositis, these two groups differed from the AI group and the control group respondents.

When BCa patients currently treated with AIs were asked during which stage of treatment their oral health had been most impacted, they reported the greatest mean impact was nearly 2 times greater when taking AI, as compared to the other treatments taken during chemotherapy (see Figure 1).

Table 4 provides an overview of oral concerns in each of the three BCa treatment groups since their BCa diagnosis. Between 20\% and 25\% of the respondents in these three groups indicated that they had a problem with their teeth and gums since the BCa diagnosis.

Chemotherapy and AI group respondents as compared to tamoxifen respondents were more likely to report that they believed that their oral health problems were related to their cancer treatment $(p<.01)$. BCa patients on chemotherapy were significantly more likely to be aware that their cancer treatment can affect their oral health than patients on tamoxifen $(93 \%$ vs. $55 \% ; p<.01)$ and patients on $\mathrm{AI}(93 \%$ vs. $56 \% ; p<.001)$. When asked who told them that their cancer treatment can affect their oral health, none of the respondents indicated their primary care provider and only a small percentage of respondents (7\% chemotherapy; 5\% tamoxifen; 10\% AI) reported that nurse practitioners had informed them. A significantly higher percentage of BCa patients undergoing chemotherapy were informed by their oncologist about oral health-related consequences compared to patients on tamoxifen ( $60 \%$ vs. 34\%; $p<.001$ ) and patients on AI $(60 \%$ vs. $32 \%$; $p<.001)$. Only $25 \%$ of chemotherapy patients, $24 \%$ of tamoxifen patients, and $25 \%$ of AI patients indicated that their dentist had shared with them that their cancer treatment can impact their oral health. Overall, only $2 \%$ of patients undergoing chemotherapy, but $35 \%$ of patients on tamoxifen and $44 \%$ of patients on AIs had not been informed about the impact of BCa treatment on oral health.

Under $30 \%$ of patients in each of the three groups reported that they had wanted to visit their dentist but could not do so due to their BCa treatment (chemotherapy: 29\%; tamoxifen: 22\%; AI: $24 \%$ ). When BCa respondents were asked at which time their dentists had discussed oral health-related effects of their cancer treatments, $52 \%$ of patients on AIs, $45 \%$ of patients on tamoxifen, and $16 \%$ of patients undergoing chemotherapy reported that their dentist had never discussed the potential treatment effects on their oral health. However, while nearly one in four patients undergoing chemotherapy reported that their dentist had discussed these impacts on their oral health at dental visits, a significantly lower percentage of patients on tamoxifen had been informed at that time $(24 \%$ vs. $8 \% ; p<.05)$. In addition, the data showed that only $14 \%$ of the AI group respondents and 18\% of the tamoxifen respondents had any discussion with their dentists about oral health effects of antiestrogen therapy.

Table 5 reports the BCa patients' responses to oral health-related patientprovider interactions by type of treatment. The $\mathrm{BCa}$ patients reported that their oncologists as well as oncology nurses were more likely to ask them about their oral health and about mucositis or xerostomia when undergoing chemotherapy than when being treated with tamoxifen or AIs. 
Respondents were surveyed as to whether their dentists had asked them if they thought their oral health had changed during cancer treatment, $50 \%$ of patients on tamoxifen, $44 \%$ of patients on $\mathrm{AI}$, and $23 \%$ of patients undergoing chemotherapy reported that their dentist had asked them this question about their oral health. There were no significant differences between the groups in terms of the respondents' reported confidence in asking their oncologist or oncology nurse or their dental provider about oral health problems. When asked about the respondents' perceptions of their oncologist's/nurse's oral health-related knowledge, $100 \%$ of the chemotherapy group respondents versus only $89 \%$ of the tamoxifen group $(p<.05)$ and $95 \%$ of the AI group respondents $(p<.05)$ thought that these providers were knowledgeable. In contrast, only $70 \%$ of BCa patients undergoing chemotherapy indicated that their dental provider was knowledgeable about oral health problems related to $\mathrm{BCa}$ treatment (chemotherapy vs. tamoxifen vs. AI: 70\% vs. $65 \%$ vs. $53 \% ; p<.05)$. In addition, $65 \%$ of patients undergoing chemotherapy versus only $56 \%$ of patients in the tamoxifen group and $52 \%$ of patients in the AI group were confident/very confident that their dental provider could answer questions related to oral healthrelated issues of cancer patients.

\section{Discussion}

This study assessed BCa patients in three different treatment conditions and a control group of healthy postmenopausal women regarding their subjective oral health, oral health-related symptoms, dental health behaviors, and communication with health care providers. The data show that while BCa patients had high dental utilization, they nevertheless had serious oral health concerns and symptoms. Another important finding is that BCa patients, especially those on antiestrogen medications, did not consider their dentists to be knowledgeable about oral healthrelated effects of cancer treatments and were not confident in their dental care providers to answer their oral health/ cancer treatment-related concerns.

Regarding the comparability of the three BCa patient groups and the control group, it is important to note that the control group respondents were on average 7 years older and thus more likely to be retired-which might have contributed to having a lower income and being less likely to have dental insurance than the BCa patients. These differences might explain why the control group respondents were less likely to have had a dental visit in the recent past, and had poorer self-reported mean dental health and periodontal health than the BCa patients. However, it should not have affected how often they engaged in oral hygienerelated behavior. It is therefore surprising that the control group subjects brushed on average less frequently than the $\mathrm{BCa}$ patients. The finding that BCa patients had on average better subjective dental and periodontal health than the control respondents could therefore not only be related to their higher dental care utilization, but also to their improved oral hygiene behavior. However, one additional explanation for these improved subjective dental health reports of $\mathrm{BCa}$ patients could be an argument provided by Taichman et al. These authors also found that women with a $\mathrm{BCa}$ diagnosis perceived their oral health more positively than women without such a diagnosis. ${ }^{29}$ They explained this finding by arguing that women with a potentially life-threatening cancer diagnosis might consider oral health-related problems as less important and thus evaluate their seriousness lower.

While BCa patients had on average better subjective dental and periodontal health than the control respondents, they reported more frequent oral symptoms such as mouth sores/mucositis, glossadynia, dysgeusia, and xerostomia within the last 3 months than the control group respondents. An analysis of the differences in the average frequencies of reported symptoms among the three BCa groups helps to understand the treatment specific concerns. BCa patients undergoing chemotherapy reported more frequent mouth sores/ mucositis, glossadynia, xerostomia, and dysgeusia than the control group respondents, but they also reported more glossadynia and dysgeusia than patients on tamoxifen and more mouth sores/ mucositis, glossadynia, and dysgeusia than patients on AIs. The only symptom reported on average less frequently by patients undergoing chemotherapy compared to patients on AIs and the control group patients is teeth sensitivity to hot, cold, or sweets. A comparison of the responses of $\mathrm{BCa}$ patients on tamoxifen versus AIs showed that there were no significant differences between these two groups.

The high frequency of oral impacts replicated previous results with patients undergoing chemotherapy ${ }^{15,16}$ and offer more insight into the subjective oral health perceptions of AI users. However, given that there is relatively little known about the oral health-related effects of BCa patients on tamoxifen, ${ }^{25}$ the findings that BCa patients on tamoxifen reported more mouth sores/mucositis and glossadynia as a consequence of their treatment than the control group respondents is definitely noteworthy and deserves future research efforts.

Regarding the oral health-related symptoms of AI users, the data showed that a higher percentage of these patients reported xerostomia than $\mathrm{BCa}$ patients undergoing chemotherapy (38\% vs. $21 \%$; $p<.05)$ and the control group respondents (38\% vs. $20 \%$; $p<.05)$. Given that saliva is vital to overall oral health because a lack of saliva is associated with increased caries and other oral health impacts, ${ }^{20,21}$ this finding needs to be communicated to health care providers interacting with BCa patients on AIs and should be followed up in future studies.

An additional sub group analysis of only the responses of $\mathrm{BCa}$ patients currently on AIs concerning the oral health impact during each stage of treatment provides additional insights into the effects of AIs on patients' oral health. Figure 1 clearly shows that the average sum of oral health impacts, namely of mouth sores/mucositis, teeth sensitive to hot, cold, or sweets, glossadynia, dry 
mouth, change in taste, bleeding gums, aching in mouth, teeth, or jaw, and bad breath was highest during current use of AIs and not during the times in which they had undergone chemotherapy or were on tamoxifen.

Another important finding of this study was the responses related to providers' oral health-related behaviors and the patients' perceptions of the providers' oral health knowledge. BCa patients indicated that oncologists/nurses did ask about oral health issues. This finding is in contrast to previous literature showing that oral health is often overlooked at the oncology visit. ${ }^{30}$ However, given that BCa survivors reported that oncologists were most likely to discuss oral health-related issues with patients undergoing chemotherapy, educational efforts may be needed to inform them that patients in this subjective survey undergoing AI therapy reported even more severe oral health consequences than patients undergoing chemotherapy.

Interestingly, low percentage of $\mathrm{BCa}$ patients reported that dentists had asked them about oral health-related effects of their cancer treatments at the various stages of treatment. Furthermore, not all BCa patients were confident/very confident that dental care providers can answer their oral health/cancer treatment-related concerns, with patients on AIs showing the least confidence (chemotherapy 70\%; tamoxifen 65\%; AI 53\%) and considering them as least knowledgeable(chemotherapy 65\%; tamoxifen 56\%; AI 52\%) about oral health-related effects of cancer treatments. While these findings merely reflect patients' subjective perceptions of their dental care providers' knowledge and communication efforts, they should nevertheless be taken as an indication that future research is needed concerning dental care providers' expertise and professional behavior in this domain. These findings along with previous results concerning dental hygienists' lack of awareness of the oral impacts of $\mathrm{AI}^{23}$ point to the urgency to increase educational efforts for dental care providers in general.
Patient-provider communication deserves additional attention because $\mathrm{BCa}$ patients should receive interprofessional care in which all providers such as oncologists, nurse practitioners, primary care physicians, and dental care providers, namely dentists and dental hygienists, collaborate and assure that $\mathrm{BCa}$ patients receive the preventive, diagnostic, and treatment-related oral health care that will allow them to maintain good oral health and have excellent oral health-related quality of life. Assuring that medical and dental school curricula inform future providers about these issues and offering continuing education courses about these topics is therefore crucial. Additional patient education efforts are needed as well.

This study had several limitations. One potential limitation is a primary reliance on self-reported data. Future research could include oral examinations to assess, for example, the degree to which patients suffered from xerostomia or mucositis. However, the questionnaire measures were pilot tested and had previously been validated. Another limitation is the fact that budgetary restrictions only allowed to conduct a study with a sample size suited to detect medium to large effects. Yet, given the importance of this topic, future research should aim to collect data from larger samples to allow the identification of even small effect sizes plus comparisons of subgroups of patients in each treatment condition. For example, while we did not collect data concerning previous cancer treatments, it would be helpful to compare responses of patients on AIs who had been previously treated with tamoxifen versus those who had not undergone such treatment in future research. Additionally, we did not consider the effects of prior exposure to bisphosphonates on BCa patients' subjective oral health and oral health concerns. Finally, our sample was not ethnically diverse. Future research should include sufficient number of patients from diverse backgrounds.

In conclusion, this study carefully documented that BCa patients undergoing chemotherapy, or being on tamoxifen and AI frequently experience serious oral health concerns. The finding that $\mathrm{BCa}$ patients reported that medical and dental care providers did not routinely inform them about potential treatment-related oral health issues and ask them about current oral health concerns calls for increased educational efforts to assure that these providers have the knowledge to provide the best possible care for these patients in the future. Research should also investigate whether these findings are applicable to cancer patients from diverse backgrounds, individuals with metastasized disease, and cancer patients at other points in the treatment trajectory.

\section{Acknowledgements}

This project was funded through a grant from Colgate. We want to thank the respondents for participating in this research.

\section{References}

1. American Cancer Society. Breast cancer facts $\varepsilon$ figures 2015-2016. Atlanta, GA. Available from https://www.cancer.org/research/ cancer-facts-statistics/breast-cancer-facts -figures.html. Accessed December 5th, 2017.

2. Carlson RW, Brown E, Burstein HJ, et al. NCCN task force report: adjuvant therapy for breast cancer. J Natl Compr Canc Netw 2006;4(Suppl 1):S1-26.

3. Carlson RW, Moench S, Hurria A, et al. NCCN task force report: breast cancer in the older woman. J Natl Compr Canc Netw 2008;6(Suppl 4):S1-25.quiz S26-7.

4. Early Breast Cancer Trialist's Collaborative Group (EBCTCG). Effects of chemotherapy and hormonal therapy for early breast cancer on recurrence and 15-year survival: an overview of the randomised trials. Lancet 2005;365(9472):1687-717.

5. Bedard PL, Cardoso F. Can some patients avoid adjuvant chemotherapy for early-stage breast cancer? Nat Rev Clin Oncol

2011;8(5):272-9.

6. Piccart-Gebhart MJ. New developments in hormone receptor-positive disease. Oncologist 2010;15(Suppl 5):18-28. 
7. Harvey JM, Clark GM, Osborne CK, Allred DC. Estrogen receptor status by immunohistochemistry is superior to the ligand-binding assay for predicting response to adjuvant endocrine therapy in breast cancer. J Clin Oncol 1999;17(5):1474-81.

8. Rastelli F, Crispino S. Factors predictive of response to hormone therapy in breast cancer. Tumori 2008;94(3):370-83.

9. Zhao H, Hei N, Wu Y, et al. Initiation of and adherence to tamoxifen and aromatase inhibitor therapy among elderly women with ductal carcinoma in situ. Cancer 2017;123(6):940-47.

10. Dunnwald LK, Rossing MA, Li CI. Hormone receptor status, tumor characteristics, and prognosis: a prospective cohort of breast cancer patients. Breast Cancer Res 2007;9(1):R6.

11. Jensen SB, Mouridsen HT, Bergmann OJ, Reibel J, Brunner N, Nauntofte B. Oral mucosal lesions, microbial changes, and taste disturbances induced by adjuvant chemotherapy in breast cancer patients. Oral Surg Oral Med Oral Pathol Oral Radiol Endod 2008;106(2):217-26.

12. Raber-Durlacher JE, Elad S, Barasch A. Oral mucositis. Oral Onco 2010;46(6):452-6.

13. Al-Ansari S, Zecha JA, Barasch A, de Lange J, Rozema FR, Raber-Durlacher JE. Oral mucositis induced by anticancer therapies. Curr Oral Health Rep 2015;2(4):202-11.

14. Barroso-Sousa R, Santana IA, Testa L, deMelo Gagliato D, Mano MS. Biological therapies in breast cancer: common toxicities and management strategies. Breast 2013;22(6):1009-18.
15. Sonis ST, Fey EG. Oral complications of cancer therapy. Oncology 2002;16(5):680-6.

16. Yuan A, Sonis S. Emerging therapies for the prevention and treatment of oral mucositis. Expert Opin Emerg Drugs 2014;19(3):343-51.

17. Taichman LS, Inglehart MR, Giannobile WV, Braun T, Kolenic G, VanPoznak C. Periodontal health in women with earlystage postmenopausal breast cancer newly on aromatase inhibitors: a pilot study. J Clin Periodontol 2015;86(7):906-16.

18. Eagle I, Benavides E, Eber R, et al. Periodontal health in breast cancer patients on aromatase inhibitors versus postmenopausal controls: a longitudinal analysis. J Clin Periodontol 2016;43(8):659-67.

19. Taichman LS, Van Poznak CH, Inglehart MR. Self-reported oral health and quality of life of postmenopausal breast cancer survivors on aromatase inhibitors and women without cancer diagnoses: a longitudinal analysis. Support Care Cancer 2016;24(11):4815-24.

20. Mosel DD, Bauer RL, Lynch DP, Hwang ST. Oral complications in the treatment of cancer patients. Oral Dis 2011;17(6):550-9.

21. Streckfus CF, Baur U, Brown LJ, Bacal C, Metter J, Nick T. Effects of estrogen status and aging on salivary flow rates in healthy Caucasian women. Gerontology 1998;44(1):32-9.

22. Saad F, Brown JE, Van Poznak C, et al. Incidence, risk factors, and outcomes of osteonecrosis of the jaw: integrated analysis from three blinded active-controlled phase III trials in cancer patients with bone metastases. Ann Oncol 2012;23(5):1341-7.

23. Migliorati CA, Epstein JB, Abt E, Berenson JR. Osteonecrosis of the jaw and bisphosphonates in cancer: a narrative review. Nat Rev Endocrinol 2011;7(1):34-42.

24. Sahin S, Karatas F, Sever AR, Altundag K. Current management of aromatase inhibitorinduced arthralgia. J BUON 2016;21(1):17-20.

25. Taichman LS, Gomez G, Inglehart MR. Oral health-related complications of breast cancer treatment: assessing dental hygienists' knowledge and professional practice. J Dent Hyg 2015;89(Suppl 2):22-37.

26. Epstein JB, Thariat J, Bensadoun RJ, et al. Oral complications of cancer and cancer therapy: from cancer treatment to survivorship. CA Cancer J Clin 2012;62(6):400-22.

27. Epstein JB, Parker IR, Epstein MS, Gupta A, Kutis S, Witkowski DM. A survey of national cancer institute-designated comprehensive cancer centers' oral health supportive care practices and resources in the USA. Support Care Cancer 2007;15(4):357-62.

28. Epstein JB, Parker IR, Epstein MS, Stevenson-Moore P. Cancer-related oral health care services and resources: a survey of oral and dental care in Canadian cancer centres. J Can Dent Assoc 2004;70(5):302-4.

29. Taichman LS, Griggs JJ, Inglehart MR. Periodontal health, perceived oral health, and dental care utilization of breast cancer survivors. J Pub Health Dent 2015;75(2):14856.

30. Rathbone EJ, Brown JE, Marshall HC, et al. Osteonecrosis of the jaw and oral healthrelated quality of life after adjuvant zoledronic acid: an adjuvant zoledronic acid to reduce recurrence trial subprotocol (BIG01/04). J Clin Oncol 2013;31(21):2685-91. 